\title{
Demographic and Socioeconomic Factors for Renouncing Further Active Therapy for Patients with Brain Metastasis of Non-Small Cell Lung Cancer
}

\author{
Gyuseo Jung ${ }^{1}$, Seok-Hyun Kim², Tae Gyu Kim³, Young Zoon Kim ${ }^{1}$ \\ 'Division of Neuro-Oncology and Department of Neurosurgery, Samsung Changwon Hospital, Sungkyunkwan University School of Medicine, \\ Changwon, Korea \\ 2Division of Hematology and Medical Oncology, Department of Internal Medicine, Samsung Changwon Hospital, Sungkyunkwan University \\ School of Medicine, Changwon, Korea \\ ${ }^{3}$ Department of Radiation Oncology, Samsung Changwon Hospital, Sungkyunkwan University School of Medicine, Changwon, Korea
}

\author{
Received February 25, 2019 \\ Revised July 12, 2019 \\ Accepted September 3, 2019 \\ Correspondence \\ Young Zoon Kim \\ Division of Neuro-Oncology \\ and Department of Neurosurgery, \\ Samsung Changwon Hospital, \\ Sungkyunkwan University \\ School of Medicine, \\ 158 Paryong-ro, Masanhoewon-gu, \\ Changwon 51353, Korea \\ Tel: +82-55-233-5241 \\ Fax: +82-55-233-8070 \\ E-mail: yzkim@skku.edu
}

Background As patients with brain metastasis (BM) of non-small cell lung cancer (NSCLC) have dismal prognosis, some of them decide to discontinue further treatment for BM. The objective of this study was to determine factors for renouncing further active therapy in patients with BM of NSCLC, focusing on their demographic and socioeconomic status.

Methods Medical records of 105 patients with radiological diagnosis of BM of NSCLC for the recent 11 years at authors' institution were retrospectively reviewed. Clinical features as well as demographic and socioeconomic characteristics such as marriage status, cohabiting family members, religious affiliations, educational background, and economic responsibility were reviewed.

Results Median overall survival (OS) was 13.84 (95\% Cl: 10.26-17.42) years in 67 patients (group A) who underwent active treatment (radiotherapy and/or chemotherapy) and 4.76 (95\% Cl: $3.12-6.41$ ) years in 38 patients (group B) who renounced active treatment. Less patients were unmarried $(p=0.046)$, more cohabitating family members $(p=0.008)$, and economically independent $(p=0.014)$ in group A than those in group B. Similarly, the unmarried, and none cohabitating family members had short OS (5.17 and 7.38 years, respectively). In multivariate analysis for predisposing factors of OS in these patients, the following demographic and socioeconomic factors had independent significance: marriage status and cohabitating family members.

Conclusion This study suggests that demographic and socioeconomic status as well as clinical factors could influence the decision of further active treatment and prognosis of patients with BM of NSCLC.

Key Words Brain metastasis; Prognosis; Socioeconomics; Non-small cell lung cancer; Palliative care; Demography.

\section{INTRODUCTION}

Lung cancer is the second most commonly diagnosed cancer in men and women. It is the most common cause of cancer death in both men and women around the world, accounting

\footnotetext{
This is an Open Access article distributed under the terms of the Creative Commons Attribution Non-Commercial License (https://creativecommons.org/licenses/by-nc/4.0) which permits unrestricted non-commercial use, distribution, and reproduction in any medium, provided the original work is properly cited.

Copyright $\odot 2019$ The Korean Brain Tumor Society, The Korean Society for NeuroOncology, and The Korean Society for Pediatric Neuro-Oncology
}

for up to $13 \%$ of all cancer deaths in women and $23 \%$ of all cancer deaths in men [1]. The incidence and mortality of lung cancer continue to rise worldwide [2,3]. The lung is recently expected to be the most common cancer site in Korea [4]. Specially, non-small cell lung cancer (NSCLC) makes up approximately $85 \%$ to $90 \%$ of all lung cancer cases [1]. Metastatic disease is diagnosed at some point in approximately $57 \%$ of all patients with NSCLC [5]. Among those with metastasis of NCSLC, 30$50 \%$ of patients will be diagnosed with brain metastases (BMs) during their disease, with rising frequency because of the avail- 
ability of novel imaging techniques and improved survival rates [6].

In fact, BMs are the most common tumors of the central nervous system $(\mathrm{CNS})$ with significant morbidity and mortality $[7,8]$ as well as devastating neurologic complications of systemic cancers [9]. BMs are reported to occur initially in $20 \%$ of patients with NSCLC [7], 10-20\% with advanced NSCLC [10], 40-50\% in those with stage III lung adenocarcinoma [11], 20-40\% in those with anaplastic lymphocyte kinase $(A L K)$-rearranged NSCLCs [11], and 45-70\% in those with ALK-rearranged NSCLCs who have been pretreated with an appropriate tyrosine kinase inhibitor [10]. Although the treatment for patients with BMs of NSCLC depends largely on the prognosis and a balance between potential benefit of the treatment modality versus known risks to the patient [12], the outcome is still uniformly dismal. For example, patients who are provided the best supportive care due to their poor condition, including systemic corticosteroids, have a median overall survival (OS) of $<2$ months at best. For patients with multiple BMs, whole brain radiation therapy (WBRT) is a traditional standard of care. Stereotactic radiosurgery (SRS) such as gamma knife radiosurgery is also an effective option when there are a limited number of metastases [13-15]. Overall, radiotherapy is effective in controlling symptoms associated with BMs [16]. However, it is difficult for patients to survive longer than 7 months after diagnosis of BM [12]. Unlike other metastatic sites of cancers, BMs can make survival short due to decreased performance status of cancer patients [17]. These neurological sequelae make it hard to treat patients more comprehensively. Therefore, many patients with BM of systemic cancer and their family members are reluctant to let them receive further treatment. Besides these medical conditions, other factors can influence the decision of discontinuing treatment for patients. However, few studies have focused on non-medical condition such as socioeconomic and demographic factors for renouncing further treatment.

Thus, the primary objective of this study was to compare socioeconomic and demographic features between patients determining to receive further active treatment for $\mathrm{BM}$ and those renouncing further treatment to estimate the prognostic role of these factors in patients with BM of NSCLC. This study also evaluated OS in patients with BM of NSCLC, determined prognostic factors influencing OS in patients with BM of NSCLC, and validate results according to known prognostic factors.

\section{MATERIALS AND METHODS}

A retrospective study was conducted of patients with radiological diagnosis of BM from NSCLC based on MRI at our institute from January 2007 to December 2017. Their NSCLCs were histopathologically confirmed at the time of diagnosis of
BM or before. After diagnosis of BM of NSCLCs, our multidisciplinary team explained the prognosis and outcome of the disease to the patients based on the literatures and began to establish the plans to treat patients according to the National Comprehensive Cancer Network (NCCN) guideline under considering patient's condition after conversion with patients and their family members. However, some patients and/or their family chose not to undergo our planned active treatment due to several reasons.

On the base of their choice, patients who underwent active treatment were categorized as group $\mathrm{A}$, and those who renounced active treatment for the disease were categorized as group B. Patients in group A decided to receive active therapeutic modalities such as surgical resection and subsequent adjuvant radiotherapy and chemotherapy if possible. On the contrary, patients in group B decided to receive mainly supportive care including corticosteroid, hyperosmolar, analgesics, and antiepileptic medication.

Thus, all patients with BM of NSCLC during the period were recruited in this retrospective study. They were analyzed in terms of patient's clinical features, tumor's radiological features, demographic characteristics, and socioeconomic status using medical records including nursing charts.

\section{Clinical assessment of patients}

Analysis was performed using clinical data of patients in group A who received active treatment and those of patients in group B who chose supportive treatment. Clinical variables included age, gender, Karnofsky Performance Scale (KPS) score, status of NSCLC, interval between the time of diagnosis of BM and NSCLC, Recursive Partitioning Analysis (RPA) class at the time of diagnosis and Graded Prognostic Assessment (GPA) score. In this study, KPS scores were determined as described by Karnofsky et al. [18], whereby patients with a score of 70 or more were capable for caring for themselves and patients with a score of less than 70 required assistance to conduct activities of daily life. RPA class was determined using the modified Radiation Therapy Oncology Group (RTOG) method [19] and GPA score was assessed based on age, KPS, extracranial metastasis, and number of BMs using the method of the largest data [20].

\section{Radiological features of BM and NSCLC}

Brain lesion parameters examined included number of enhanced mass in the brain and time of diagnosis of BM. The number of BM was counted as the mass with enhancement with gadolinium in T1 weighted MRI. In terms of the interval between the time of BM and NSCLC, BM diagnosed $\leq 2$ months from NSCLC diagnosis was considered synchronous and that diagnosed $>2$ months from the time of NSCLC diagnosis was 
defined as metachronous. Extracranial metastasis was estimated by abdominal and chest CT scan as well as positron emission tomography (PET)-CT performed at the time of diagnosis of BMs. The status of NSCLC was determined as stable in the metachronous metastasis if there was no interval change of the cancer on the radiographical study. It was considered unstable in the metachronous metastasis if there was growth of the cancer and for all cases of synchronous metastasis.

\section{Demographic and socioeconomic characteristics of patients}

Family history of cancer was examined. Marriage status was classified as married or unmarried. In addition, cohabiting family members were identified and 1st generation offspring were separately categorized as sons and daughters. Among those family members, those who had economic responsibility for treatment were also identified. Social factors included patient religious affiliations and educational backgrounds.

\section{Survival analysis and statistical analysis}

Medical records of clinical history, demographic and socioeconomic history, family environment, and radiographic reports of all study subjects were analyzed. Date of death was confirmed and recorded. OS was defined as the time from date of diagnosis of BM till death. The date of brain biopsy, if performed, or the date of MRI study was recorded as the date of diagnosis.

Statistical analyses were performed using SPSS ver. 20.0 (IBM Corp., Armonk, NY, USA). Differences between subgroups were analyzed with Student's t-test for normally distributed continuous values and Mann-Whitney U-test for nonnormally distributed continuous values. Chi-square test was used to analyze categorical variables. OS was calculated according to the Kaplan-Meier method. Comparisons among groups were performed with log-rank tests. Variables that were significantly associated with OS of NSCLC patients with BM in univariate analyses were examined in multivariate analysis. Several additional variables associated with OS in the literature were also included in the multivariate analysis. In this analysis, Cox proportional hazards regression model was used to assess independent effects of specific factors on OS and define hazard ratios of significant covariates. Two-sided $p$-values $<0.05$ were considered statistically significant.

\section{Ethical statement}

The Institutional Review Board (IRB) of Sungkyunkwan University Samsung Changwon Hospital approved the study protocol (IRB number: SCMC 2018-12-006). All studies were conducted according to guidelines of the Declaration of Helsinki for biomedical research. Informed consent was waived due to its retrospective nature.

\section{RESULTS}

\section{Clinical features of patients}

Among a total 121 patients with BM of NSCLC, our study ultimately enrolled 105 patients with BM of NSCLC. Sixteen patients were excluded from this study because of insufficient medical data. Of these 105 patients, 67 (group A) received active treatment for the BM of NSCLC, and 38 (group B) renounced active treatment but received supportive care.

Mean age of all patients was 67.9 years (64.1 years in group $A$ and 74.6 years in group $B ; p=0.024$ ). It ranged from 34.5 to 91.2 years (34.5 to 91.2 years in group $A$ and 54.3 to 87.0 years in group B) (Table 1). There were 57 males and 48 females. Sixty-one (58.1\%) patients had a relatively good performance with KPS $\geq 70$. Fifteen (14.3\%) patients were categorized as RPA class I and 26 (24.7\%) patients were categorized as RPA class III. Twenty-one (20.0\%) patients had GPA score $0-1$. Forty-seven (44.8\%) patients had GPA class 1.5-2.5. Twenty-nine (27.6\%) patients had GPA score 3. Eight (7.6\%) patients had GPA score 3.5-4.0. The detail clinical data showing comparative results between group A and B was summarized in Table 1.

Major therapeutic modalities of BM were surgical resection in 23 (34.3\%) patients, WBRT alone in 16 (23.8\%) patients, SRS alone in 5 (7.5\%) patients, WBRT plus SRS in 17 (25.4\%) patients, and salvage chemotherapy in $6(9.0 \%)$ patients (Table 1).

\section{Radiologic characteristics of BM and NSCLC}

Fifty (47.6\%) patients had multiple BMs of number $>3$. Eightythree $(79.0 \%)$ patients had extracranial metastases. Sixty-five (61.9\%) patients had stable NSCLC. Thirty-three (31.4\%) BMs were found at the same time as NSCLC. The detail radiological data showing comparative results between group $\mathrm{A}$ and $\mathrm{B}$ was summarized in Table 1.

\section{Demographic and socioeconomic environment of patients with BM of NSCLC}

Forty-seven (44.8\%) patients had family history of cancer. In group A, 61 (91.0\%) of 67 patients were married. Of these, $43(64.2 \%)$ patients lived with their spouses. In group B, 33 (86.8\%) of 38 patients were married. Of these, $10(26.3 \%)$ lived with their spouses. The proportion of patients living with their spouses was significantly less in group B $(p=0.046)$ (Table 2$)$. Only 9 (13.5\%) patients lived alone in group A. However, 21 $(55.3 \%)$ patients had no cohabitating family members in group $\mathrm{B}(p=0.008)$. Especially, as many as 44 patients $(65.7 \%)$ in group A lived with their son and/or daughter and spouses while only 2 (5.2\%) patients in group B did ( $p=0.008)$. Forty-one (61.2\%) patients in group A had an independent economic capability to pay for the medical care while only $7(18.4 \%)$ patients in group $\mathrm{B}$ had such capability ( $p=0.014)$. Additionally, multiple 1st gen- 
Table 1. Clinical characteristics of the patients with BM of NSCLC

\begin{tabular}{|c|c|c|c|c|}
\hline & Total $(n=105)$ & Group A $(n=67)$ & Group B $(n=38)$ & $p$ value \\
\hline Mean age (years) & $67.9(34.5-91.2)$ & $64.1(34.5-91.2)$ & $74.6(54.3-87.0)$ & 0.024 \\
\hline Male:female & $57: 48$ & $35: 32$ & $22: 16$ & 0.486 \\
\hline KPS & & & & 0.017 \\
\hline$<70$ & $44(41.9)$ & $24(35.8)$ & $20(52.6)$ & \\
\hline$\geq 70$ & $61(58.1)$ & $43(64.2)$ & $18(47.4)$ & \\
\hline Number of BM & & & & 0.651 \\
\hline Single & $27(25.7)$ & $19(28.4)$ & $8(21.1)$ & \\
\hline Oligometastasis (2-3) & $28(26.7)$ & $17(25.4)$ & $11(28.9)$ & \\
\hline Multiple (>3) & $50(47.6)$ & $31(46.3)$ & $19(50.0)$ & \\
\hline Extracranial metastasis & & & & 0.073 \\
\hline Yes & $83(79.0)$ & $49(73.1)$ & $34(89.5)$ & \\
\hline No & $22(21.0)$ & $18(26.9)$ & $4(15.8)$ & \\
\hline Status of NSCLC & & & & 0.708 \\
\hline Stable & $65(61.9)$ & $42(62.7)$ & $23(60.5)$ & \\
\hline Unstable & $40(38.1)$ & $25(37.3)$ & $15(40.5)$ & \\
\hline Time interval of BM & & & & 0.041 \\
\hline Synchronous ( $\leq 2$ months) & $33(31.4)$ & $27(40.3)$ & $6(15.8)$ & \\
\hline Metachronous ( $>2$ months) & $72(68.6)$ & $40(59.7)$ & $32(74.2)$ & \\
\hline RPA class & & & & 0.009 \\
\hline I & $15(14.3)$ & $15(22.4)$ & $0(0.0)$ & \\
\hline II & $64(61.0)$ & $45(67.2)$ & $19(50.0)$ & \\
\hline III & $26(24.7)$ & $7(10.4)$ & $19(50.0)$ & \\
\hline GPA score & & & & 0.004 \\
\hline $0-1$ & $21(20.0)$ & $17(25.4)$ & $4(10.5)$ & \\
\hline $1.5-2.5$ & $47(44.8)$ & $38(56.7)$ & $9(23.7)$ & \\
\hline 3.0 & $29(27.6)$ & $10(14.9)$ & $19(50.0)$ & \\
\hline $3.5-4.0$ & $8(7.6)$ & $2(3.0)$ & $6(15.8)$ & \\
\hline \multicolumn{5}{|l|}{ Major treatment of BM } \\
\hline Surgical resection & $23(21.9)$ & $23(34.3)$ & - & \\
\hline WBRT alone & $16(15.2)$ & $16(23.8)$ & - & \\
\hline SRS alone & $5(4.8)$ & $5(7.5)$ & - & \\
\hline WBRT plus SRS & $17(16.2)$ & $17(25.4)$ & - & \\
\hline Salvage chemotherapy & $6(5.7)$ & $6(9.0)$ & - & \\
\hline
\end{tabular}

Variables are presented as mean (range) or number (\%). BM, brain metastasis; GPA, Graded Prognostic Assessment; KPS, Karnofsky Performance Scale; NSCLC, non-small cell lung cancer; RPA, Recursive Partitioning Analysis; SRS, stereotactic radiosurgery; WBRT, whole brain radiotherapy

eration offspring shared the fee of medical care for $17(25.4 \%)$ patients in group A and 6 (15.8\%) patients in group B. However, in the case that only one son or daughter of the patient bore totally the cost of medical care, there were more patients $(n=17$, $44.7 \%)$ in group B than those $(n=3,4.5 \%)$ in group A. There was no significant difference in religious affiliation or educational background between groups A and B (Table 2). The detail demographic and socioeconomic data showing comparative results between group A and B was summarized in Table 2 .

\section{Follow-up and OS of patients}

Follow-up duration ranged from 1.56 months to 18.37 months.
During the follow-up period, 73 (69.5\%) patients died. The median length of OS was 10.56 months [95\% confidence interval (CI): 7.13-14.21 months]. The median OS of patients who were treated with active therapeutic modality for BM (group A) was 13.84 months (95\% CI: 10.26-17.42 months). The median OS of patients who renounced the further active treatment for BM (group B) was 4.76 months (95\% CI: 3.12-6.41 months) $(p<0.001)$ (Table 3).

\section{Univariate analysis for predisposing factors of OS of patients with BM of NSCLC}

In terms of clinical factors, the following variables were sig- 
Table 2. Summaries of demographic and socioeconomic environment of the patients

\begin{tabular}{|c|c|c|c|}
\hline & Group A $(n=67)$ & Group B $(n=38)$ & $p$ value \\
\hline Family history of cancer & & & 0.031 \\
\hline Yes & $18(26.9)$ & $29(76.3)$ & \\
\hline No & $49(73.1)$ & $9(23.7)$ & \\
\hline Marriage status & & & 0.046 \\
\hline The married and living with spouse & $43(64.2)$ & $10(26.3)$ & \\
\hline The married and living without spouse & $18(26.9)$ & $23(60.5)$ & \\
\hline The divorced after marriage & $7(10.4)$ & $6(15.8)$ & \\
\hline The bereaved after marriage & $11(16.5)$ & $17(44.7)$ & \\
\hline The unmarried & $6(8.9)$ & $5(13.2)$ & \\
\hline Cohabiting family members & & & 0.008 \\
\hline None & $9(13.5)$ & $21(55.3)$ & \\
\hline 1st generation offspring & $6(8.9)$ & $6(15.8)$ & \\
\hline Life partner alone & $8(11.9)$ & $9(23.7)$ & \\
\hline 1st generation offspring+life partner & $44(65.7)$ & $2(5.2)$ & \\
\hline Religious affiliations & & & 0.274 \\
\hline None & $21(31.3)$ & $24(63.1)$ & \\
\hline Buddhism & $15(22.4)$ & $5(13.2)$ & \\
\hline The Christian religion & $17(25.4)$ & $5(13.2)$ & \\
\hline Roman Catholicism & $14(20.9)$ & $4(10.5)$ & \\
\hline Educational background & & & 0.069 \\
\hline Uneducated & $3(4.5)$ & $10(26.3)$ & \\
\hline Elementary graduate & $14(20.9)$ & $15(39.5)$ & \\
\hline Middle school graduate & $16(23.9)$ & $6(15.8)$ & \\
\hline High school graduate & $20(29.8)$ & $3(7.9)$ & \\
\hline University graduate & $14(20.9)$ & $4(10.5)$ & \\
\hline Economic capability for medical care & & & 0.014 \\
\hline Patient oneself & $41(61.2)$ & $7(18.4)$ & \\
\hline Single 1st generation offspring & $3(4.5)$ & $17(44.7)$ & \\
\hline Son & $1(1.5)$ & $12(31.6)$ & \\
\hline Daughter & $3(3.0)$ & $5(13.1)$ & \\
\hline Multiple 1st generation offspring & $17(25.4)$ & $6(15.8)$ & \\
\hline Parents, brother or sister & $6(8.9)$ & $5(13.2)$ & \\
\hline Charity organization & $0(0.0)$ & $3(7.9)$ & \\
\hline
\end{tabular}

Variables are presented as number (\%).

nificantly associated with longer OS: age $<65$ years (vs. $\geq 65$ years; $p=0.048$ ), KPS $\geq 70$ (vs. $<70 ; p=0.001$ ), stable NSCLC (vs. unstable NSCLC; $p=0.019$ ), synchronous metastasis (vs. metachronous metastasis; $p=0.042$ ), RPA class I (vs. class III; $p<$ 0.001 ), GPA score $3.5-4.0$ (vs. score of $0-1 ; p<0.001$ ), and active treatment (vs. conservative treatment; $p<0.001$ ) (Table 3 ). However, gender, number of BMs, or extracranial metastasis was not significantly associated with OS in univariate analysis.

In terms of demographic and socioeconomic factors, the following variables were significantly associated with longer OS: absence of family history of cancer (vs. presence; $p=0.018$ ), married but living without spouse (vs. unmarried; $p=0.007$ ), married and living spouse (vs. unmarried; $p=0.002$ ), cohabitation with 1 st generation offspring without spouse (vs. none; $p=0.047$ ), cohabitation with 1st generation offspring with spouse together (vs. none; $p=0.011$ ), independent economic capability for medical care (vs. dependent economic capability for medical care on single 1st generation offspring; $p=0.045$ ), and economic capability for medical care by direct family members such as parents, brothers, and sisters (vs. independent economic capability for medical care; $p=0.038$ ) (Table 4 ). However, religious affiliation or educational background was not associated with OS in univariate analysis.

\section{Multivariate analysis for predisposing factors of OS of patients with BM of NSCLC}

Multivariate analysis using the Cox proportional hazard regression model for survival demonstrated that the following fac- 
Table 3. Univariate analysis for clinical predisposing factors of OS in the patients

\begin{tabular}{|c|c|c|c|}
\hline Clinical factors & Median OS $(95 \% \mathrm{CI})$ & Hazard ratio $(95 \% \mathrm{CI})$ & $p$ value \\
\hline \multicolumn{4}{|l|}{ Age (years) } \\
\hline$\geq 65$ & $9.47(7.56-11.38)$ & 1.00 & \\
\hline$<65$ & $12.39(9.25-15.53)$ & $2.21(1.13-3.29)$ & 0.048 \\
\hline \multicolumn{4}{|l|}{ Gender } \\
\hline Male & $10.21(7.98-12.44)$ & 1.00 & \\
\hline Female & $10.96(8.36-13.56)$ & $1.27(0.69-1.85)$ & 0.805 \\
\hline \multicolumn{4}{|l|}{ KPS } \\
\hline$<70$ & $7.32(5.68-8.96)$ & 1.00 & \\
\hline$\geq 70$ & $12.89(9.71-16.07)$ & $4.36(2.52-6.21)$ & 0.001 \\
\hline \multicolumn{4}{|l|}{ Number of BM } \\
\hline Multiple & $9.02(6.82-11.22)$ & 1.00 & \\
\hline Single+oligometastasis & $11.95(9.05-14.85)$ & $1.84(0.83-2.85)$ & 0.346 \\
\hline \multicolumn{4}{|l|}{ Extracranial metastasis } \\
\hline Yes & $10.11(8.14-12.08)$ & 1.00 & \\
\hline No & $12.23(9.37-15.09)$ & $1.62(0.88-2.36)$ & 0.482 \\
\hline \multicolumn{4}{|l|}{ Status of NSCLC } \\
\hline Unstable & $7.76(6.21-9.31)$ & 1.00 & \\
\hline Stable & $12.27(10.19-14.35)$ & $3.51(1.74-5.28)$ & 0.019 \\
\hline \multicolumn{4}{|l|}{ Time interval of BM } \\
\hline Metachronous (>2 months) & $9.33(7.28-11.38)$ & 1.00 & \\
\hline Synchronous ( $\leq 2$ months) & $13.21(9.98-163.44)$ & $2.46(1.29-3.63)$ & 0.042 \\
\hline \multicolumn{4}{|l|}{ RPA class } \\
\hline III & $5.76(4.49-7.03)$ & 1.00 & \\
\hline II & $10.42(7.64-13.21)$ & $3.08(1.54-4.62)$ & 0.026 \\
\hline I & $19.43(15.62-23.24)$ & $5.37(2.80-7.94)$ & $<0.001$ \\
\hline \multicolumn{4}{|l|}{ GPA score } \\
\hline $0-1$ & $3.92(3.12-4.72)$ & 1.00 & \\
\hline $1.5-2.5$ & $8.97(7.22-10.72)$ & $2.74(1.56-3.92)$ & 0.037 \\
\hline 3.0 & $15.45(11.13-19.77)$ & $4.26(2.63-5.89)$ & 0.002 \\
\hline $3.5-4.0$ & $20.38(15.62-25.14)$ & $7.58(4.63-10.53)$ & $<0.001$ \\
\hline \multicolumn{4}{|l|}{ Major treatment of BM } \\
\hline Conservative treatment & $4.76(3.12-6.41)$ & 1.00 & \\
\hline Active treatment & $13.84(10.26-17.42)$ & $9.43(5.71-13.05)$ & $<0.001$ \\
\hline
\end{tabular}

BM, brain metastasis; CI, confidence interval; GPA, Graded Prognostic Assessment; KPS, Karnofsky Performance Scale; NSCLC, non-small cell lung cancer; OS, overall survival; RPA, Recursive Partitioning Analysis

tors were independently associated with longer OS: age $<65$ years (vs. $\geq 65$ years, $95 \%$ CI: $1.28-3.42 ; p=0.046$ ), KPS $\geq 70$ (vs. $<70,95 \%$ CI: 2.24-6.52; $p=0.009$ ), stable NSCLC (vs. unstable, 95\% CI: 1.85-5.89; $p=0.020$ ), RPA class I (vs. class II, 95\% CI: 1.62-4.96; $p=0.026$ and vs. class III, 95\% CI: 3.25-9.83; $p<0.001$ ), RPA class II (vs. class III, 95\% CI: 1.51-3.73; $p=0.041$ ), GPA score of 0-2.5 (vs. score of 3.0-4.0, 95\% CI: 4.08-9.39; $p<0.001$ ), active treatment (vs. conservative, 95\% CI: 5.73-13.63; $p<0.001$ ), married (vs. unmarried, 95\% CI: 1.47-4.85; $p=0.026$ ), absence of family history of cancer (vs. presence, 95\% CI: 1.43$4.19 ; p=0.038$ ), and presence of cohabiting family members (vs. absence, 95\% CI: 1.70-5.46; $p=0.017$ ) (Table 5). However, economic capability for medical care which showed significant association with OS in univariate analysis was not associated with OS in multivariate analysis. Kaplan-Meier survival curve analysis and log-rank test revealed the same results (Fig. 1).

\section{DISCUSSION}

Our retrospective study examined demographic and socioeconomic factors of patients with BM of NSCLC who renounced further active treatment for their disease. We found that family history of cancer, marriage status, composition of cohabitating family members, and economic capability for medical care seemed to have influence on the decision of renouncing further active treatment. Exclusively, a better supported family com- 
Table 4. Univariate analysis for demographic and socioeconomic predisposing factors of OS in the patients

\begin{tabular}{|c|c|c|c|}
\hline & Median OS (95\% CI) & Hazard ratio $(95 \% \mathrm{CI})$ & $p$ value \\
\hline \multicolumn{4}{|l|}{ Family history of cancer } \\
\hline Yes & $8.03(6.21-9.85)$ & 1.00 & \\
\hline No & $12.60(9.38-15.82)$ & $3.19(1.94-4.43)$ & 0.018 \\
\hline \multicolumn{4}{|l|}{ Marriage status } \\
\hline The unmarried & $5.17(3.66-6.68)$ & 1.00 & \\
\hline The married \& living without spouse & $10.62(7.40-13.84)$ & $3.88(2.41-5.35)$ & 0.007 \\
\hline The married \& living with spouse & $11.59(7.92-15.26)$ & $4.25(2.70-5.81)$ & 0.002 \\
\hline \multicolumn{4}{|l|}{ Cohabiting family members } \\
\hline None & $7.38(5.64-9.12)$ & 1.00 & \\
\hline 1st generation offspring & $9.47(7.01-11.93)$ & $2.22(1.08-3.36)$ & 0.047 \\
\hline Life partner alone & $9.26(7.13-11.39)$ & $2.07(0.97-3.17)$ & 0.062 \\
\hline 1st generation offspring+life partner & $13.68(10.46-16.89)$ & $3.67(2.29-5.05)$ & 0.011 \\
\hline \multicolumn{4}{|l|}{ Religious affiliations } \\
\hline None & $10.05(7.26-12.84)$ & 1.00 & \\
\hline Buddhism & $10.76(7.59-13.93)$ & $1.13(0.67-1.58)$ & 0.872 \\
\hline The Christian religion & $10.93(7.74-14.12)$ & $1.19(0.58-1.79)$ & 0.831 \\
\hline Roman Catholicism & $11.12(7.95-14.29)$ & $1.26(0.70-1.82)$ & 0.786 \\
\hline \multicolumn{4}{|l|}{ Educational background } \\
\hline Uneducated & $10.61(7.88-13.34)$ & 1.00 & \\
\hline Elementary graduate & $10.38(7.51-13.25)$ & $0.97(0.42-1.52)$ & 0.924 \\
\hline Middle school graduate & $10.41(6.93-13.89)$ & $0.98(0.39-1.57)$ & 0.953 \\
\hline High school graduate & $10.98(8.06-13.91)$ & $1.10(0.56-1.64)$ & 0.905 \\
\hline University graduate & $10.36(6.84-13.88)$ & $0.95(0.24-1.66)$ & 0.946 \\
\hline \multicolumn{4}{|l|}{ Economic capability for medical care } \\
\hline Patient oneself & $10.69(7.43-13.88)$ & 1.00 & \\
\hline Single 1st generation offspring & $8.27(6.16-10.38)$ & $0.64(0.29-0.98)$ & 0.045 \\
\hline Multiple 1st generation offspring & $11.65(8.71-14.59)$ & $1.85(0.87-2.83)$ & 0.091 \\
\hline Parents, brother or sister & $12.32(8.60-16.04)$ & $2.73(1.31-4.15)$ & 0.038 \\
\hline Charity organization & $10.02(7.28-12.76)$ & $0.96(0.66-1.26)$ & 0.846 \\
\hline
\end{tabular}

CI, confidence interval; OS, overall survival

Table 5. Multivariate analysis for predisposing factors of OS in the patients using Cox regression model

\begin{tabular}{lrr}
\hline & Hazard ratio (95\% CI) & $p$ value \\
\hline Age ( $\geq 65$ years vs. $<65$ years) & $2.35(1.28-3.42)$ & 0.046 \\
KPS ( $\geq 70$ vs. $<70)$ & $4.38(2.24-6.52)$ & 0.009 \\
Status of NSCLC (stable vs. unstable) & $3.87(1.85-5.89)$ & 0.020 \\
Time interval of BM (synchronous vs. metachronous) & $2.16(0.84-3.48)$ & 0.073 \\
RPA class & & 0.026 \\
$\quad$ (I vs. II) & $3.29(1.62-4.96)$ & $<0.001$ \\
$\quad$ I vs. III) & $6.54(3.25-9.83)$ & 0.041 \\
$\quad$ II vs. III) & $2.62(1.51-3.73)$ & $<0.001$ \\
GPA score (0-2.5 vs. 3.0-4.0) & $6.74(4.08-9.39)$ & $<0.001$ \\
Treatment of BM (active vs. conservative) & $9.68(5.73-13.63)$ & 0.026 \\
Marriage status (married vs. unmarried) & $3.16(1.47-4.85)$ & 0.038 \\
Family history of cancer (no vs. yes) & $2.81(1.43-4.19)$ & 0.017 \\
Cohabiting family members (presence vs. absence) & $3.58(1.70-5.46)$ & 0.102 \\
Economic capability for medical care (patient's oneself vs. other's help) & $1.92(0.88-2.96)$ & \\
\hline BM, brain metastas &
\end{tabular}

BM, brain metastasis; CI, confidence interval; GPA, Graded Prognostic Assessment; KPS, Karnofsky Performance Scale; NSCLC, non-small cell lung cancer; OS, overall survival; RPA, Recursive Partitioning Analysis 
munity system to patients, for example, more family members who help the patient emotionally and economically, makes the patient to receive further treatment for the disease more actively. It can be a unique characteristic of Korean culture for family members to support patients in all aspects of life. It is an ostensible aspect that if more family members are around the patient, more supports can be given to the patient. However, emotional support provided by more family members can play an active
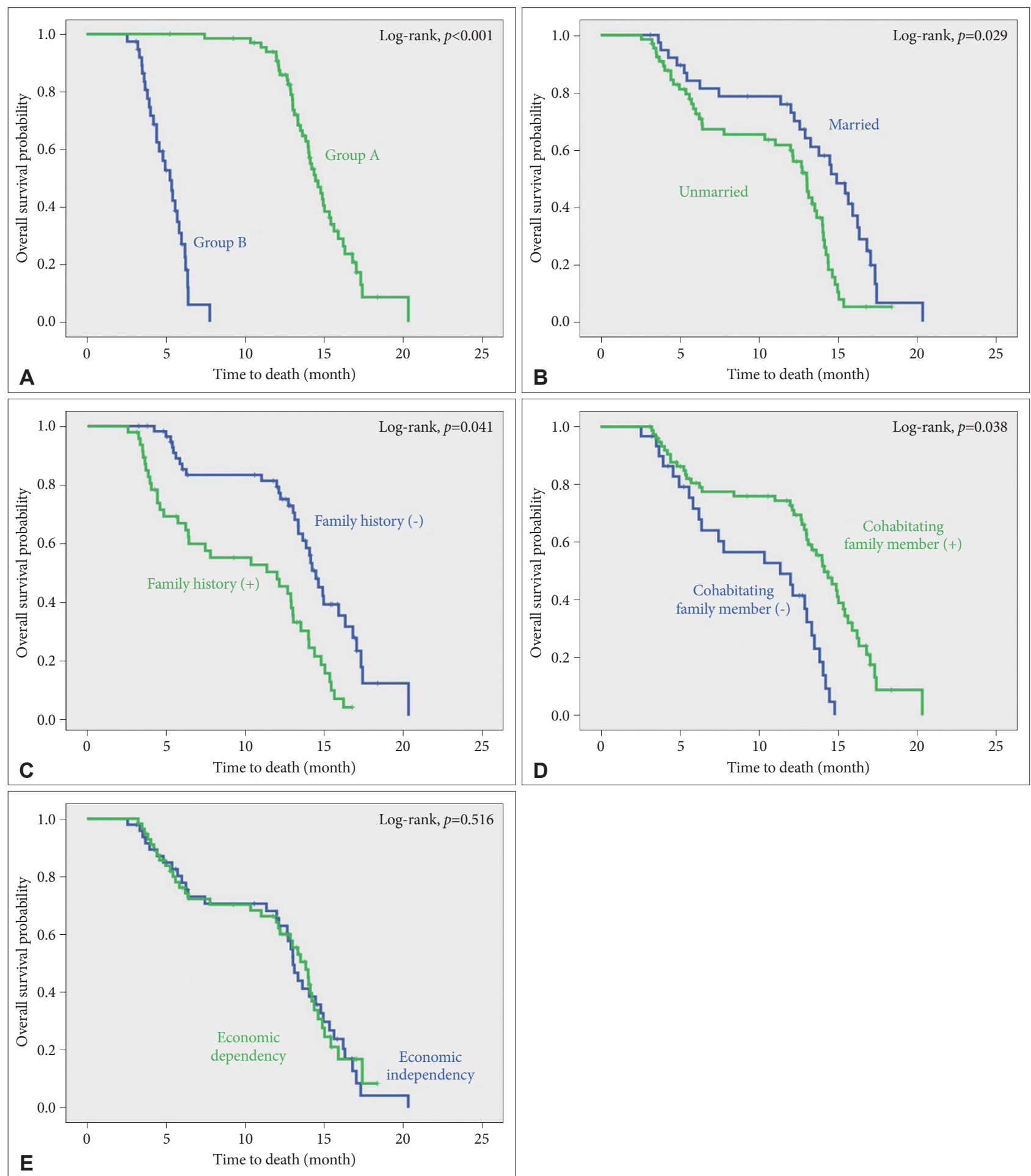

Fig. 1. Kaplan-Meier survival curves of overall survival for patients with brain metastasis of non-small cell lung cancer according to (A) groups (Group A vs. Group B), (B) marriage status (married vs. unmarried), (C) family history of cancer (presence vs. absence), (D) cohabitating family member (presence vs. absence), and (E) economic capability to pay for medical care (dependent vs. independent) 
part in the background because patients with cancer can suffer from depression. Depression is a common form of psychological distress observed in people with cancer. Most studies have reported that the prevalence of depression in oncological populations ranges from $15 \%$ to $30 \%$, with variations attributable to different screening tools and diagnostic criteria across studies [21]. Recent results support the hypothesis that a variety of psychological interventions may be effective in promoting resilience to stress and reinforcing social support, including standard cognitive behavioral therapy and mindfulness-based therapy with multimodal approaches such as psychoeducational interventions, anticipatory guidance, and measures of psychosocial support [22]. Although emotional support provided by family members is not systemic or specialized compared to psychosocial support provided by a special physician such as psychiatrist, it can be more comfortable and familiar to the patient. Additionally, dietary support can be much effective for the patient if there is rich information about their preference for the taste. Data also indicate that a diet enriched with aforementioned elements may exert protective effect against the development of depressive symptoms [23]. In fact, most replicated clinical data on dietary recommendations for the prevention of depression indicate that increased consumption of fruit, vegetables, legumes, whole-grain cereals, nuts, seeds, fish rich in omega- 3 polyunsaturated fatty acids, and limitation of the intake of processed-foods, 'fast' foods, and commercial bakery goods has benefits [23].

Other factors that can improve emotional stress include exercise and good sleep. Effective physical activity plays a vital role in improving physical fitness in cancer survivors. It is considered as a key factor of lifestyle interventions. It can also reverse negative treatment-related side effects [24]. The prevalence of poor sleep quality is common among cancer populations, affecting up to $50 \%$ of cancer survivors. It is 2 -fold or 3 -fold of that in the general community [25].

Unfortunately, the present study did not have any information on depression of enrolled patients. According to the unique Korean culture, more family members who have direct and close contact with the patient might provide more support for the patient in every aspect of the life. Thus, emotional support should be able to reduce depression of the patient. However, randomized clinical trials are mandatory to prove such hypothesis.

In terms of economic support, there was a significant difference in economic capability to pay for medical care between groups A and B. Financial burden is thought to be one of important factors affecting the decision of further active treatment in cancer patients. Although the Registry Project for Seriously Ill Patients of the Korean National Health Insurance supports $95 \%$ of medical fee for treating cancer, the cost is still too heavy burden to patients and their family. According to unpublished data of 10 patients with NSCLC at our institute, the mean cost of annual medical fee from initial step of NSCLC diagnosis to BM was 6,362,000 Korean won (KRW) (range: 3,785,000 to $14,055,000 \mathrm{KRW}$ ) that was paid by the patient. Despite there are many variations in medical condition of individual patient and the Registry Project for Seriously Ill Patients of the Korean National Health Insurance supports part of the cost, the financial burden is not a simple problem to the patient. Total amount of medical cost for patients with NSCLC (sum of patient's burden and government's coverage) was 46,278,000 KRW on average for the 10 patients with NSCLC. There was no Korean report about the economic burden on the patient in searchable database. One American study reported that the mean cost for medical care of patients with NSCLC was 184,872 US dollars (203,359,200 KRW in exchange rate of 1 dollar to 1,100 KRW) [26]. Actually, among the 41 patients with independent capability for medical care in group A, 39 (95.1\%) patients had private insurance for health to cover most of the cost. Although it is necessary for systemic approach to reduce the economic burden for medical care, hospice consultation can be considered as a way of resolution. Referral to hospice consultation may reduce the economic burden by lowering intensive care unit admission rates, lowering out-of-hospital death rates, and decreasing the use of chemotherapy within 1 month prior to death without affecting the overall survival of patients with lung cancer [27].

This study has some limitations. First, our data were analyzed based on medical records retrospectively. Thus, there is limitation when interpreting our results. As mentioned above, emotional support for patients might affect their depression. However, such data were not included as there was no information for the diagnosis of depression. Second, this was not a randomized study. Thus, it was impossible for authors to exclude bias that the treatment team might have depending on how strongly they encouraged patients to pursue active treatment. Third, the diagnosis of $\mathrm{BM}$ was based on radiological impression rather than histopathological impression. Although the incidence is thought to be low, other malignant brain tumors such as highgrade glioma or primary CNS lymphoma, not BM, might have been included in this study. Finally, as our data were based on results from a single institute in Korea, these results cannot be generalized. Data from a multi-center study should be investigated to validate our results.

Conclusively, the present study suggests that demographic and socioeconomic status such as family history of cancer, marriage status, cohabitating family members, and economic capability to pay for medical care could influence the decision of further active treatment and prognosis of patients with BM of NSCLC, in addition to clinical factors. However, this study shows a certain hypothesis that emotional support originated from 
demographic and socioeconomic status could influence the decision of further treatment for the disease by controlling depression in cancer patients. As this study was a retrospective analysis based on medical records of patients from a single institute, multi-institute and randomized clinical trials are needed in the future for validation.

\section{Conflicts of Interest}

The authors have no potential conflicts of interest.

\section{Acknowledgments}

We thank Drs. Young Min Kim and Mi-Ok Sunwoo (Department of Radiology, Samsung Changwon Hospital) for their review of radiological images, and Dr. Young Wook Kim (Department of Biostatistics, Samsung Changwon Hospital) for assistance with the statistical analysis.

\section{REFERENCES}

1. Jemal A, Bray F, Center MM, Ferlay J, Ward E, Forman D. Global cancer statistics. CA Cancer J Clin 2011;61:69-90.

2. Malvezzi M, Bertuccio P, Levi F, La Vecchia C, Negri E. European cancer mortality predictions for the year 2013. Ann Oncol 2013;24:792-800.

3. International Agency for Research on Cancer, World Health Organization. Cancer fact sheets: estimated cancer incidence, mortality and prevalence worldwide in 2012. Globocan 2012 (Accessed November 8, 2018, at: http://gco.iarc.fr/today/data/pdf/fact-sheets/cancers/cancerfact-sheets-11.pdf.)

4. Jung KW, Won YJ, Kong HJ, Lee ES. Prediction of cancer incidence and mortality in Korea, 2018. Cancer Res Treat 2018;50:317-23.

5. National Cancer Institute. Cancer stat facts: lung and bronchus cancer. Surveillance, Epidemiology and End Results Program (Accessed November 8, 2018, at: https://seer.cancer.gov.)

6. Preusser M, Winkler F, Valiente M, et al. Recent advances in the biology and treatment of brain metastases of non-small cell lung cancer: summary of a multidisciplinary roundtable discussion. ESMO Open 2018;3:e00262.

7. Barnholtz-Sloan JS, Sloan AE, Davis FG, Vigneau FD, Lai P, Sawaya RE. Incidence proportions of brain metastases in patients diagnosed (1973 to 2001) in the Metropolitan Detroit Cancer Surveillance System. J Clin Oncol 2004;22:2865-72.

8. Kohler BA, Ward E, McCarthy BJ, et al. Annual report to the nation on the status of cancer, 1975-2007, featuring tumors of the brain and other nervous system. J Natl Cancer Inst 2011;103:714-36.

9. DeAngelis LM, Posner JB. Intracranial metastases. In: DeAngelis LM, Posner JB, editors. Neurologic complications of cancer. New York: Oxford University Press; 2009. p. 141-93.

10. Toyokawa G, Seto T, Takenoyama M, Ichinose Y. Insights into brain metastasis in patients with ALK+ lung cancer: is the brain truly a sanctuary? Cancer Metastasis Rev 2015;34:797-805.

11. Mamon HJ, Yeap BY, Jänne PA, et al. High risk of brain metastases in surgically staged IIIA non-small-cell lung cancer patients treated with surgery, chemotherapy, and radiation. J Clin Oncol 2005;23:1530-7.

12. Sperduto PW, Kased N, Roberge D, et al. Summary report on the graded prognostic assessment: an accurate and facile diagnosis-specific tool to estimate survival for patients with brain metastases. J Clin Oncol 2012;30:419-25.

13. Tsao $\mathrm{MN}, \mathrm{Xu} \mathrm{W}$, Wong RK, et al. Whole brain radiotherapy for the treatment of newly diagnosed multiple brain metastases. Cochrane Database Syst Rev 2012;1:CD003869.

14. Reck M, Popat S, Reinmuth N, De Ruysscher D, Kerr KM, Peters S; ESMO Guidelines Working Group. Metastatic non-small-cell lung cancer (NSCLC): ESMO Clinical Practice Guidelines for diagnosis, treatment and follow-up. Ann Oncol 2014;25 Suppl 3:iii27-39.

15. Chang WS, Kim HY, Chang JW, Park YG, Chang JH. Analysis of radiosurgical results in patients with brain metastases according to the number of brain lesions: is stereotactic radiosurgery effective for multiple brain metastases? J Neurosurg 2010;113 Suppl:73-8.

16. Rosen LS, Gordon D, Tchekmedyian NS, et al. Long-term efficacy and safety of zoledronic acid in the treatment of skeletal metastases in patients with nonsmall cell lung carcinoma and other solid tumors: a randomized, Phase III, double-blind, placebo-controlled trial. Cancer 2004;100:2613-21.

17. Dayan A, Koca D, Akman T, Oztop I, Ellidokuz H, Yilmaz U. The factors that have an impact on the development of brain metastasis in the patients with breast cancer. J Cancer Res Ther 2012;8:542-8.

18. Karnofsky DA, Abelmann WH, Craver LF, Burchenal JH. The use of the nitrogen mustards in the palliative treatment of carcinoma. With particular reference to bronchogenic carcinoma. Cancer 1948;1:63456.

19. Li J, Wang M, Won M, et al. Validation and simplification of the Radiation Therapy Oncology Group recursive partitioning analysis classification for glioblastoma. Int J Radiat Oncol Biol Phys 2011;81:623-30.

20. Sperduto PW, Chao ST, Sneed PK, et al. Diagnosis-specific prognostic factors, indexes, and treatment outcomes for patients with newly diagnosed brain metastases: a multi-institutional analysis of 4,259 patients. Int J Radiat Oncol Biol Phys 2010;77:655-61.

21. Cardoso G, Graca J, Klut C, Trancas B, Papoila A. Depression and anxiety symptoms following cancer diagnosis: a cross-sectional study. Psychol Health Med 2016;21:562-70.

22. Zhang MF, Wen YS, Liu WY, Peng LF, Wu XD, Liu QW. Effectiveness of mindfulness-based therapy for reducing anxiety and depression in patients with cancer: a meta-analysis. Medicine (Baltimore) 2015;94: e0897.

23. Opie RS, Itsiopoulos C, Parletta N, et al. Dietary recommendations for the prevention of depression. Nutr Neurosci 2017;20:161-71.

24. Gokal K, Wallis D, Ahmed S, Boiangiu I, Kancherla K, Munir F. Effects of a self-managed home-based walking intervention on psychosocial health outcomes for breast cancer patients receiving chemotherapy: a randomised controlled trial. Support Care Cancer 2016;24:1139-66.

25. Savard J, Morin CM. Insomnia in the context of cancer: a review of a neglected problem. J Clin Oncol 2001;19:895-908.

26. Ganguli A, Henk H, Teitelbaum A, Ray S. PCN39 Burden of brain metastasis in an metastatic non-small cell lung cancer (NSCLC) population. Value Health 2011;14:A440-1.

27. Yoo SH, Keam B, Kim M, Kim TM, Kim DW, Heo DS. The effect of hospice consultation on aggressive treatment of lung cancer. Cancer Res Treat 2018;50:720-8. 